\begin{tabular}{l} 
O P E R A T I O N S R E S E A R C H A N D D E C I S I O N S \\
\hline No. 4
\end{tabular}

DOI: $10.37190 /$ ord200404

\title{
ON THE LAW OF THE ITERATED LOGARITHM IN HYBRID MULTIPHASE QUEUEING SYSTEMS
}

\author{
SAULIUS MINKEVIČIUS* \\ Institute of Data Science and Digital Technologies, Akademijos St. 4, Vilnius, LT-04812, Lithuania
}

The model of a hybrid multiphase queueing system (HMQS) has been developed to measure the performance of complex computer networks working under conditions of heavy traffic. Two probability limit theorems (laws of the iterated logarithm, LIL) are presented for a queue length of jobs in HMQS.

Keywords: operations research, model, computer network, queueing network, performance evaluation, iterated logarithm

\section{Introduction}

Whitby in his book about artificial intelligence [21] states that the human brain consists of 100 networks of networks (NoN). One of the examples of NoN is a hybrid multiphase queueing system (HMQS). At first, we present the summary of works dedicated to a particular case of HMQS and multiphase queueing system (MQS, see Fig. 1). One can apply limit theorems for a waiting time of a customer and a queue length of customers to get probabilistic characteristics of MQS under various conditions of heavy traffic $[2,3]$. The most fundamental example (a single-phase case, where the time intervals in between the arrivals of customers to MQS are independent identically distributed random variables and there is a single device, working independently of the output in heavy traffic) has been completely investigated by several authors $[2,8]$. Iglehart [5] carefully investigated a single-device case and obtained laws of the iterated logarithm (LIL) for this case. It is surprising to note that the fundamental results of Iglehart on the queueing systems, working in heavy traffic are rarely used [4-8]. There are only a few papers on the theory of MQS in heavy traffic $[10,12,13]$ with, however, no proof of LIL for the probabilistic characteristics of MQS in heavy traffic. LIL for a cumulative waiting time

*Email address: minkevicius.saulius@gmail.com

Received 22 December 2019, accepted 10 December 2020 
of a customer and a waiting time of a customer is proved in another paper [13]. In this work, theorems on LIL for other main characteristics of MQS in heavy traffic (cumulative queue length of customers, the queue length of customers, waiting time of a customer) are proved. The main tools for the analysis of MQS in heavy traffic are functional LIL for a Wiener process and a renewal process (the proof can be found in [20,5]). We submit some definitions from the theory of metric spaces [1, Chapter 2]. Let $C$ be a metric space consisting of real continuous functions in $[0,1]$ with a uniform metric

$$
\rho(x, y)=\sup _{0 \leq t \leq 1}|x(t)-y(t)|, x, y \in C
$$

Also, let $D$ be a space of all real-valued right-continuous functions in $[0,1]$ having left limits and endowed with the Skorokhod topology induced by the metric $d$ (under which $D$ is complete and separable). Define $k(\delta)$ as a set of absolutely continuous functions $x \in C$ such that $x(0)=0$ and $\int_{0}^{1}[\dot{x}(t)]^{2} d t \leq \delta^{2}$, where $\dot{x}$ is a derivative of $x$, which exists almost everywhere, according to the Lebesque measure. Strassen [20] showed that $k(\delta)$ is a compact set for $x \in k(\delta)$ and

$$
|x(b)-x(a)| \leq \delta(b-a)^{1 / 2}
$$

\section{Formulation of the problem}

At first, we define an open queueing network (OQN, see, e.g., [18]). Besides, we define OQNl for $l=1,2, \ldots, m$ as a sub-network which is a part of HMQS structure (Fig. 1). An arrival stream of customers, let us say $e_{1}(t)$, comes to HMQS, and is spread in the sub-network in the first phase OQN1 of HMQS, based on a number, e.g., $m_{1}$ of working devices, $\frac{e_{1}(t)}{m_{1}}$. The customer leaves OQN1 after service and enters the second phase OQN2, etc., until she arrives at the last phase OQNm, where she leaves the network entirely (the basic examples of HMQS and MQS are provided in Fig. 1).

We investigate an $m$ th phase HMQS (i.e., when a customer is served in the $j$ th phase of HMQS, she is routed to the $j+1$ phase of MQS, and only when the customer is served in the $m$ th phase of HMQS, she leaves the system entirely). Let us denote by $t_{n}$ a time of arrival of the $n$th customer to the first OQN1; $S_{l, n}^{(j)}$ denotes the service time of the $n$th customer in the $l$ th node of the $j$ th OQN of HMQS; $z_{n}=t_{n+1}-t_{n}$, denotes also $z_{j, l}$ a time of arrival of the $n$th customer in the $l$ th node of the $j$ th OQN of HMQS. Let us introduce mutually independent renewal processes $x_{l, j}(t)=\max \left\{k: \sum_{i=1}^{k} S_{l, i}^{(j)} \leq t\right\}$ (a total number 
of customers that can be served in the $j$ th node of the lth OQN of HMQS until time $t$ (presuming that all the devices are working without time waste), $e(t)=\max \left\{k: \sum_{i=1}^{k} z_{j, l} \leq t\right\}$ (the total number of customers that arrive at the $l$ th OQN of HMQS until time moment $t$ ). Next, denote by $\tau_{l, j}(t)$ the total number of customers that depart from the $j$ th node of the $l$ th OQN of the HMQS until time $t ; Q_{l, j}(t)$ a queue length of customers of the $j$ th node in the $l$ th OQN of HMQS at the time moment $t ; v_{l, j}(t)$ $=\sum_{i=1}^{j} Q_{l, j}(t)$ as a cumulative queue length of customers until the $j$ th node of the OQN of HMQS at the time moment $t, l=1,2, \ldots, m, j=1,2, \ldots, k_{j}$, and $t>0$. Suppose that the queue length of customers in each node of each OQN in HMQS is unlimited, the service discipline of customers is "first come, first served" (FCFS). All random variables are defined in one common probability space $(S, F, P)$. Consider interarrival times at HMQS $\left(z_{n}\right)$ and service times $\left(S_{l, n}^{(j)}\right)$ in each node of OQN in HMQS for $l=1,2, \ldots, m, j=1,2, \ldots, k_{j}$ as mutually independent identically distributed random variables.

Let us define

$$
\begin{gathered}
\beta_{l, j}=\left(E S_{l, 1}^{(j)}\right)^{-1}, \quad \beta_{0}=\left(E z_{1}\right)^{-1}, \quad \alpha_{l, j}=\beta_{0}-\beta_{l, j}, \quad \alpha_{l, 0}=0, \hat{\sigma}_{l, j}^{2}=D S_{l, 1}^{(j)}\left(E S_{l, 1}^{j}\right)^{-3}>0 \\
\hat{\sigma}_{0}^{2}=D z_{1}\left(E z_{1}\right)^{-3}>0, \quad \tilde{\sigma}_{l, j}^{2}=\hat{\sigma}_{l, 0}^{2}+\hat{\sigma}_{l, j}^{2}, \quad \sigma_{l, j}^{2}=\hat{\sigma}_{l, j}^{2}+\hat{\sigma}_{l, j-1}^{2} \\
\hat{x}_{l, j}(t)=e(t)-x_{l, j}(t), \quad l=1,2, \ldots, m, \quad j=1,2, \ldots, k_{j}, t \geq 0
\end{gathered}
$$

Assume the following condition to be fulfilled $\beta_{l, 0}>\beta_{l, 1}>\ldots>\beta_{l, j}>0$, then

$$
\alpha_{l, j}>\alpha_{l, j-1}>\ldots>\alpha_{l, 0}>0, \text { where } l=1,2, \ldots, m, j=1,2, \ldots, k_{j}
$$

One of the main results of the paper is a theorem on LIL for the cumulative length of customers in HMQS under conditions of heavy traffic.

Theorem 1. If conditions (1) are fulfilled, then

$$
P\left(\varlimsup_{n \rightarrow \infty} \frac{v_{l, j}(t)-\alpha_{l, j} t}{\tilde{\sigma}_{l, j} a(t)}=1\right)=1 \text { and } P\left(\lim _{t \rightarrow \infty} \frac{v_{l, j}(t)-\alpha_{l, j} t}{\tilde{\sigma}_{l, j} a(t)}=-1\right)=1
$$

for $l 1,2, \ldots, m, j=1,2, \ldots, k_{j}$ and $a(t)=\sqrt{2 t \ln t}, t>0$. 
Proof. First of all, denote

$$
\begin{aligned}
& \left.p_{l, j}=1-\sum_{i=1}^{k_{j}} p_{l, i}, \quad p_{l, j}^{t}=1-\sum_{i=1}^{k_{j}} p_{l, i}^{t}, \quad w_{l, j}(t)=x_{l, j}(t)\left(\sum_{i=1}^{k_{j}}\left|p_{l, i}^{t}-p_{l, i}\right|\right)\right) \\
& \gamma_{l, j}(t)=\sup _{0 \leq s \leq t}\left(x_{l, j}(s)-\tau_{l, j}(s)\right), \quad \hat{x}_{l, j}(t)=x_{l, j}(t) p_{\gamma_{j}}, \quad \overline{\hat{x}}_{l, j}(t)=e_{l}(t)-\hat{x}_{l, j}(t) \\
& l=1,2, \ldots, m, \quad j=1,2, \ldots, k_{j}, t \geq 0
\end{aligned}
$$

Note that

$$
\begin{aligned}
\hat{\tau}_{l, j}(t) & =\tau_{l, j}(t)-\sum_{i=1}^{k_{j}} \tau_{l, i}(t)=\tau_{l, j}(t)\left(1-\sum_{i=1}^{k_{j}} \frac{\tau_{l, i}(t)}{\tau_{l, j}(t)}\right)=\tau_{l, j}(t)\left(1-\sum_{i=1}^{k_{j}} p_{l, i}^{t}\right) \\
& =\tau_{l, j}(t) p_{l, j}^{t}, l=1,2, \ldots, m, j=1,2, \ldots, k_{j}, t \geq 0
\end{aligned}
$$

Applying the results of the paper [14], we obtain that

$$
\left|\hat{\tau}_{l, j}(t)-\hat{x}_{l, j}(t)\right| \leq w_{l, j}(t)+\gamma_{l, j}(t), \quad l=1,2, \ldots, m, j=1,2, \ldots, k_{j}, t \geq 0
$$

In Minkevičius [12] relations

$$
\begin{gathered}
Q_{l, j}(t)=\hat{\tau}_{l, j-1}(t)-\hat{\tau}_{l, j}(t) \\
\left.Q_{l, j}(t)=f_{t} \overline{\hat{\tau}}_{l, j-1}(\cdot)-\hat{x}_{l, j}(\cdot)\right) \\
Q_{l, j}(t)=f_{t}\left(\overline{\hat{x}}_{j}(\cdot)-\sum_{i=1}^{j-1} Q_{l, i}(\cdot)\right), f_{t}(x(\cdot))=x(t)-\inf _{0 \leq s \leq t} x(s) \\
l=1,2, \ldots, m, j=1,2, \ldots, k_{j}, t \geq 0
\end{gathered}
$$

Now, we present another estimate of inequality (4)

$$
\begin{aligned}
& \hat{x}_{l, j}(t)-\hat{\tau}_{l, j}(t)=\sup _{0 \leq s \leq t}\left(\hat{x}_{l, j}(s)-\hat{\tau}_{l, j-1}(s)\right)=\sup _{0 \leq s \leq t}\left(\hat{x}_{l, j}(s)-\hat{\tau}_{l, j-1}(s)\right) \\
& +\sup _{0 \leq s \leq t}\left(\hat{x}_{l, j-1}(s)-\hat{\tau}_{l, j-2}(s)\right) \leq \sum_{i=1}^{k}\left(\sup _{0 \leq s \leq t}\left(\hat{x}_{l, i}(s)-\hat{x}_{l, i-1}(s)\right)\right)=\sum_{i=1}^{k}\left(\sup _{0 \leq s \leq t}\left(\overline{\hat{x}}_{l, i-1}(s)-\overline{\hat{x}}_{l, i}(s)\right)\right) \\
& l=1,2, \ldots, m, j=1,2, \ldots, k_{j}, t \geq 0
\end{aligned}
$$


From (8) we derive

$$
\begin{aligned}
v_{j}(t) & =\sum_{i=1}^{j} Q_{l, i}(t)=\sum_{i=1}^{j}\left\{\overline{\hat{\tau}}_{l, i-1}(t)-\hat{\tau}_{l, i}(t)\right\}=e_{l}(t)-\hat{\tau}_{l, j}(t)=e_{l}(t)-\hat{x}_{l, j}(t)+\hat{x}_{l, j}(t)-\tau_{l, j}(t) \\
& \leq \overline{\hat{x}}_{l, j}(t)+\sum_{i=1}^{k}\left\{\sup _{0 \leq s \leq t}\left(\overline{\hat{x}}_{l, i-1}(s)-\overline{\hat{x}}_{l, i}(s)\right)\right\}, \quad l=1,2, \ldots, m, j=1,2, \ldots, k_{j}, t \geq 0
\end{aligned}
$$

Since for any $l\left(l=1,2, \ldots, m, j=1,2, \ldots, k_{j}\right)$

$$
v_{l, j}(t)=e_{l}(t)-\tau_{l, j}(t) \geq e_{l}(t)-\hat{x}_{l, j}(t)=\overline{\hat{x}}_{l, j}(t) \geq \overline{\hat{x}}_{l, j}(t)-\sum_{i=1}^{k}\left\{\sup _{0 \leq s \leq t}\left(\hat{\bar{x}}_{l, i-1}(s)-\overline{\hat{x}}_{l, i}(s)\right)\right\}
$$

we find from (5) the following estimate

$$
\left|v_{l, j}(t)-\overline{\hat{x}}_{l, j}(t)\right| \leq \sum_{i=1}^{k}\left\{\sup _{0 \leq s \leq t}\left(\overline{\hat{x}}_{l, i-1}(s)-\overline{\hat{x}}_{l, i}(s)\right)\right\}
$$

Suppose

$$
\begin{aligned}
& v_{l, j}^{n}(t)=\frac{v_{l, j}(n t)-\alpha_{l, j} n t}{a(n)}, \quad \text { and } x_{l, j}^{n}(t)=\frac{e(n t)-\hat{x}_{l, j}(n t)-\alpha_{l, j} n t}{a(n)} \\
& l=1,2, \ldots, m, j=1,2, \ldots, k_{j}, t \geq 0
\end{aligned}
$$

By Corollary 2.1 in [9] for any fixed $j$ and $l\left\{x_{l, j}^{n}, n \geq 1\right\}$, there is a relatively compact set in $(\mathcal{D}, D)$, and the set of its limit points coincides with $k\left(\tilde{\sigma}_{l, j}\right)$. Then, in view of inequality (8), the family $\left\{v_{l, j}^{n}(t), n \geq 3\right\}$ is also a relatively compact set, and the set of its limit points coincides with $k\left(\tilde{\sigma}_{l, j}\right)$.

Hence, we prove that

$$
\begin{aligned}
& P\left(\varlimsup_{n \rightarrow \infty} \frac{v_{l, j}(t)-\alpha_{l, j} t}{\tilde{\sigma}_{l, j} a(t)}=1\right)=1, \text { and } P\left(\frac{\lim }{t \rightarrow \infty} \frac{v_{l, j}(t)-\alpha_{l, j} t}{\tilde{\sigma}_{l, j} a(t)}=-1\right)=1 \\
& l=1,2, \ldots, m, j=1,2, \ldots, k_{j}, t \geq 0
\end{aligned}
$$

The proof is complete. 
The theorem on LIL for the queue length of customers in HMQS is proved similarly as Theorem 1 .

Theorem 2. If conditions (1) are fulfilled, then

$$
\begin{aligned}
& P\left(\varlimsup_{t \rightarrow \infty} \frac{Q_{l, j}(t)-\left(\alpha_{l, j}-\alpha_{l, j-1}\right) t}{\sigma_{l, j} a(t)}=1\right)=1, \text { and } P\left(\lim _{t \rightarrow \infty} \frac{Q_{l, j}(t)-\left(\alpha_{l, j}-\alpha_{l, j-1}\right) t}{\sigma_{l, j} a(t)}=-1\right)=1 \\
& l=1,2, \ldots, m, j=1,2, \ldots, k_{j}, t \geq 0
\end{aligned}
$$

Proof. It follows from (7) that

$$
\begin{aligned}
& \left|Q_{l, j}(t)-\left(\hat{x}_{l, j}(t)-\hat{x}_{l, j-1}(t)\right)\right| \leq\left|v_{l, j}(t)-\hat{x}_{l, j}(t)\right|+\left|v_{l, j-1}(t)-\hat{x}_{l, j-1}(t)\right| \\
& \leq 2\left\{\sum_{i=1}^{k} \sup _{0 \leq s \leq t}\left(\overline{\hat{x}}_{l, i-1}(s)-\overline{\hat{x}}_{l, i}(s)\right)\right\}, \quad l=1,2, \ldots, m, j=1,2, \ldots, k_{j}, t \geq 0
\end{aligned}
$$

Define a family of random functions as

$$
\begin{aligned}
& Q_{l, j}^{n}(t)=\frac{Q_{l, j}(n t)-\left(\alpha_{l, j}-\alpha_{l, j-1}\right) n t}{a(n)} \\
& \tilde{x}_{l, j}^{n}(t)=\frac{\overline{\hat{x}}_{l, j}(n t)-\overline{\hat{x}}_{l, j-1}(n t)-\left(\alpha_{l, j}-\alpha_{l, j-1}\right) n t}{a(n)} \\
& l=1,2, \ldots, m, j=1,2, \ldots, k_{j}, t \geq 0, n \geq 3
\end{aligned}
$$

Further proof of Theorem 2 is analogous to that of Theorem 1. The proof is complete. A graphical illustration is provided for hybrid MQS (on the left hand side) and generic MQS.

Remark 1. The results of [5] in a single-device case follow from Theorem 2. As the results in the paper of Sakalauskas, Minkeviius [19] on the open queueing network.

Remark 2. In this work, a recursive proof method is used, which was previously used in the study of multiphase queueing systems (see [16]) and open queueing networks (see [17]).

Remark 3. The right side of Fig. 1 shows a diagram of the multiphase queueing system (it has the $k$ phase) and the left side of Fig. 1 shows a diagram of the hybrid multiphase system (instead of phases there is an open queueing network, the common system also has a $k$ phase). 


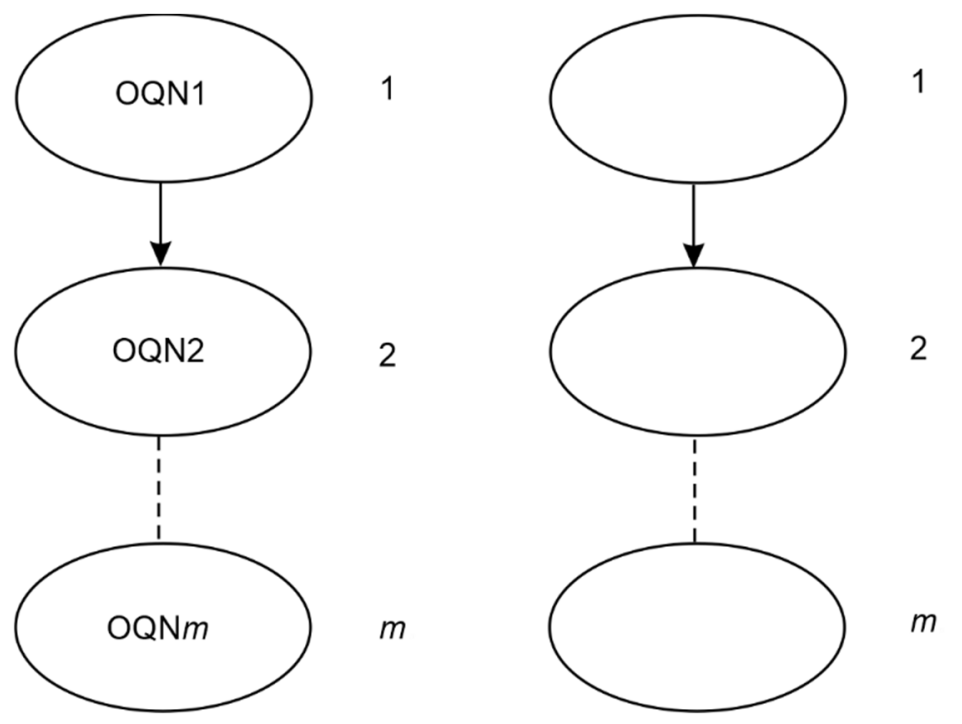

Fig. 1. Hybrid multiphase queueing system (left), multiphase queueing system (right)

\section{Concluding remarks}

The theorems of this work are proved for a class of HMQS in heavy traffic with reference to the principle first come, first served, endless waiting time of a customer in each phase of the queue, when times among customers arriving to HMQS are independent identically distributed random variables. However, similar limit theorems can be applied to a wider class of HMQS in heavy traffic: as the arrival and service of customers in a queue are by group, and times between the customers arriving to HMQS are independent and weakly dependent random variables, etc.

\section{References}

[1] BiLlingSLey P., Convergence of probability measures, Nauka, Moscow 1977, 352 (2), 137-168 (in Russian).

[2] Borovkov A.A., Probability Processes in the Queueing Theory, Nauka, Moscow 1972, 300 (in Russian).

[3] Borovkov A.A., Asymptotic Methods of the Queueing Theory, Nauka, Moscow 1980, 420 (in Russian).

[4] IGLEHART D.L., Limiting diffusion approximations for many queues and the repairman problem, J. Appl. Prob., 1965, 2, 429-441.

[5] IgLehart D.L., Multiple channel queues in heavy traffic. IV. Law of the iterated logarithm, Zeits. Wahrs. Theory, 1971, 17, 168-180.

[6] IgLeHART D.L., Functional limit theorems for the GI/G/1 queue in light traffic, Adv. Appl. Prob., 1971, 3, 269-281.

[7] Iglehart D.L., Extreme values in the GI/G/1 queue in light traffic, Ann. Math. Stat., 1972, 43, 627-635.

[8] IGLeHART D.L., Weak convergence in queueing theory, Adv. Appl. Prob., 1973, 5, 570-594. 
[9] Iglehart D.L., Whitt W., Multiple channel queues in heavy traffic. I, Adv. Appl. Prob., 1970, 2, $150-177$.

[10] IGLEhART D.L., WhitT W., Multiple channel queues in heavy traffic. II. Sequences, networks and batches, Adv. Appl. Prob., 1970, 2, 355-369.

[11] KyPRIANOU E., The virtual waiting time of the GI/G/1 queue in heavy traffic, Adv. Appl. Prob., 1974, 3, 249-269.

[12] MinKeVIČIUS S., Transient phenomena in multiphase queues, Liet. Mat. Rin., 1991, 31 (1), 136-145.

[13] MinkEVIČIUS S., On the law of the iterated logarithm in multiphase queues, Liet. Mat. Rin., 1995, 35 (1), 360-365.

[14] MinKeviČIUS S., On the law of the iterated logarithm in multiserver open queueing networks, Stochastics, 2014, 86, 46-59.

[15] MinKEVIČIUS S., On the analysis of the law of the iterated logarithm in open queueing networks, Int. J. Comp. Math., Comp. Syst. Theory, 2019, 4 (2), 76-94.

[16] MinkeVIČIUS S., Fluid limits for the waiting time of a customer in multiphase queues, 2019, https: //elib.bsu.by/bitstream/123456789/233376/1/249-252.pdf

[17] MinKeviČIUS S., GreIČIUS E., Heavy Traffic Limits for the Extreme Waiting Time in Multiphase Queueing Systems, Meth. Comp. Appl. Prob., 2019, 21 (1), 109-124.

[18] ReIman M., Open queueing networks in heavy traffic, Math. Oper. Res., 1984, 9, 441-459.

[19] SAKAlAUSKAS L., MinKEVIČIUS S., On the law of the iterated logarithm in open queueing networks, Eur. J. Oper. Res., 2000, 120, 632-640.

[20] Strassen V., An invariance principle for the law of the iterated logarithm, Zeits. Wahrs. Theory, 1964, 3, 211-226.

[21] WhitBy B., Artificial Intellgence. A beginners guide, Oneworld, Oxford 2004.

[22] Wнiтt W., Heavy traffic limit theorems for queues. A survey, Lecture Notes in Economics and Mathematical Systems, 98, Springer-Verlag, Berlin 1974, 307-350. 